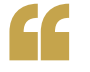

\section{SHC interacts with ERK to negatively regulate its} kinase activity

\title{
CELL SIGNALLING
}

\section{SHC keeps ERK under control}

SHC (SH2 domain-containing transforming protein) is well known as an adaptor protein that binds to active receptor Tyr kinases (RTKs) to promote downstream signalling events, including the activation of ERK. Suen et al. now show that, in the absence of growth factors, SHC interacts with ERK to negatively regulate its kinase activity and keep basal signalling in check.

While investigating the protein binding properties of SHC, the authors observed that it interacted with ERK in mammalian cells. SHCERK interactions were reduced upon growth factor-mediated activation of epidermal growth factor receptor (EGFR) - an RTK that binds SHC. A detailed analysis of this interaction, using structural, biophysical and

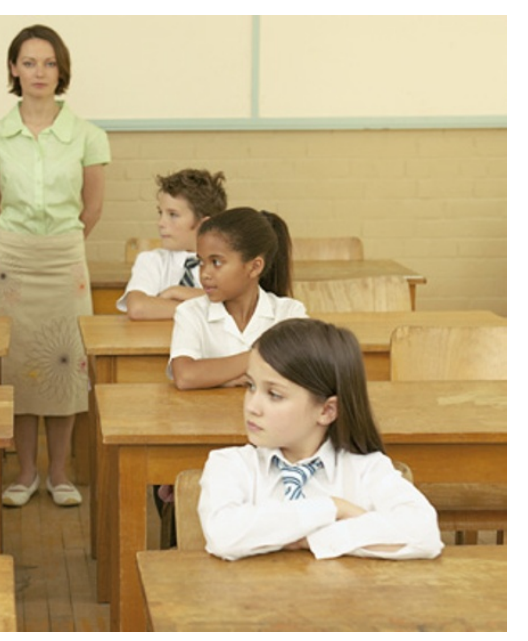

functional approaches, revealed that a long loop within the phosphotyrosine-binding (PTB) domain of SHC (termed SHC3R) interacted with the amino-terminal lobe of ERK. Arg98 in SHC3R was essential for SHC-ERK binding, as this interaction was abolished when Arg98 was mutated. Thus, SHC interacts with ERK in the absence of growth factor stimulation.

What is the physiological relevance of this, perhaps unexpected, interaction? In the absence of growth factors, the level of phosphorylated ERK was increased in cells expressing SHC that cannot bind ERK ( $\mathrm{SHC}^{\mathrm{R} 98 \mathrm{Q}}$ ) compared with cells expressing wildtype SHC. Furthermore, depleting cells of SHC using short hairpin RNA increased the level of phosphorylated and nuclear ERK. Therefore, SHC seems to reduce the phosphorylation, and thus the activity, of ERK by directly interacting with this kinase.

Next, the authors assessed whether SHC also negatively regulates ERK in vivo in Caenorhabditis elegans. During C. elegans germline development, ERK activation is biphasic; its downregulation between two regions of activation in the gonad allows the normal meiotic progression of oogenic germ cells. In C. elegans that lack shc-1, ERK expression was continuous and resulted in a phenotype similar to that seen for let-60 gain-of-function mutants (LET-60, the worm homologue of mammalian RAS, is an upstream activator of ERK). Overexpressing human wild-type SHC, but not $\mathrm{SHC}^{\mathrm{R} 98 \mathrm{Q}}$, restored biphasic ERK expression in worms. Thus, SHC also interacts with, and negatively regulates, ERK in vivo.

Finally, the authors asked what triggers the dissociation of SHCERK complexes to allow signalling in response to growth factors. They found that a Tyr-phosphorylated peptide, pTRKA, which is derived from the TRKA RTK that binds SHC, prevented SHC-ERK interactions, as did the phosphorylated cytoplasmic domain of EGFR. The phosphorylated ligand interacts with the PTB of SHC at a site distal from its ERK interaction site. Thus, the binding of phosphorylated ligand to the PTB of SHC promotes SHC-ERK dissociation through a conformational change within the domain.

This study reveals that, in addition to its established role in triggering ERK activity by stimulating upstream kinases in response to growth factors, SHC interacts with ERK to keep it in check under basal conditions and prevent inappropriate signalling. Katharine H. Wrighton

ORIGINAL RESEARCH PAPER Suen, K. M. et al. Interaction with Shc prevents aberrant Erk activation in the absence of extracellular stimuli. Nature Struct. Mol. Biol. 14 Apr 2013 (doi:10.1038/ nsmb.2557) 